\title{
Implementación del sistema de pastoreo racional Voisin en la finca La Gloria del municipio de Puerto López (Meta)
}

Implementation of rational grazing system Voisin in the farm La Gloria in the municipality of Puerto Lopez (Meta)

\author{
Reina D. Carlos ${ }^{1}$; Pachón, Héctor A. ${ }^{2}$ y Sánchez Vladimir ${ }^{3}$ \\ ${ }^{1}$ MVZ.; ${ }^{2}$ MV. y ${ }^{3}$ MVZ. MSc. Docente Universidad de los Llanos \\ vladimirsanchez@unillanos.edu.co
}

Recibido 20 de Enero 2012, aprobado 22 de Febrero 2012

\section{RESUMEN}

Este trabajo se realizó en la finca la Gloria de la vereda Melúa del municipio de Puerto López, en el departamento del Meta, la cual está situada a 300 msnm, con una temperatura de $30^{\circ} \mathrm{C}$, una humedad relativa de $82 \%$, los suelos que predominan en la región son francoarenosos y ácidos, y con una topografía de $60 \%$ plana, $30 \%$ ondulada y un $10 \%$ quebrada. Los índices de producción de la finca la Gloria en especial el de las ganancias de peso se mantuvieron bajos en un sistema de pastoreo tradicional, por lo tanto, este proyecto consistió en implementar un sistema de pastoreo racional en la finca el cual permitió optimizar el consumo del forraje sin pérdidas significativas, de igual manera, contribuyó en el mejoramiento de los índices de producción de la finca, entre otros beneficios. Se realizó un diagnóstico inicial para conocer el estado de la finca y como se encontraba en términos de suelo, forraje que se ofrecía al ganado, fuentes de agua y demás recursos que permitan determinar en qué condiciones se iba de ejecutar el proyecto. Se planificó con base en el diagnóstico inicial cuales eran las mejores condiciones para la realización del proyecto, al igual, que un presupuesto de acuerdo a lo aprobado entre las partes interesadas en la ejecución. Se ejecutó el proyecto de implementación del sistema de pastoreo racional Voisin de acuerdo a lo establecido y aprobado por parte del productor y se organizó el sistema de pastoreo actual para ayudar en el mejoramiento de los índices de producción 
(ganancia de peso) en el ganado bovino de la finca. Se logro pasar de producir en 64 hectáreas 21 toneladas de carne a 52 toneladas en la misma área, es decir, 0.3 toneladas de carne por hectárea a 0.8 toneladas de carne por hectárea, además se concientizó a los empleados de la finca la Gloria en buenas prácticas ganaderas y biosanitarias y lo relacionado con el correcto manejo de las praderas.

Palabras clave: Pastoreo rotacional voisin, ganancia de peso, bovinos de ceba.

\begin{abstract}
This work was done on the farm La Gloria of the village Melua municipality of Puerto Lopez, Meta department, which is situated at 300 meters above sea level, with a temperature of $30{ }^{\circ} \mathrm{C}$, relative humidity of $82 \%$, soil prevailing in the region are loam and acid, with a $60 \%$ flat topography, $30 \%$ rolling and $10 \%$ broken. Production rates of the farm La Gloria especially the weight gains were low in a traditional grazing system, therefore, this project was to implement a rational grazing system on the farm which helped to optimize the use without significant loss of forage, likewise, contributed in improving production rates of the estate, among other benefits. Initial diagnosis was performed to check the status of the farm and as it was in terms of soil, which provided fodder for livestock, water sources and other resources to determine what conditions are going to implement the project. Was planned based on the initial diagnosis which were the best conditions for the project, as well, that a budget as approved by the parties concerned in the execution. It implemented the project of implementation of sound grazing system Voisin according to established and approved by the producer and organized the current grazing system to assist in improving production rates (weight gain) in cattle of the estate. Spending was achieved in 64 acres of producing 21 tons of meat to 52 tons in the same area, that is, 0.3 tons of meat per hectare to 0.8 tons of meat per hectare, in addition to the employees became aware of the farm La Glory in good farming practices and life sciences and related to the proper management of pastures.
\end{abstract}


Keywords: Voisin rotational grazing, weight gain, cattle fattening.

\section{INTRODUCCIÓN}

El productor de ganado bovino en Colombia ha comenzado a tener una transformación cultural y de pensamiento con respecto a la ganadería, que aunque ha sido a pasos cortos, se ha iniciado a contemplar la explotación ganadera como empresa, y es ahí cuando estamos siendo llamados todos los profesionales del sector pecuario a ser líderes en esta conversión de pensamiento que en ultimas beneficia a los productores, comercializadores y sin duda alguna a los profesionales en el área de la salud y producción de la ganadería colombiana.

El Pastoreo Racional Voisin constituye un método eficaz para el rescate de la sostenibilidad de la producción ganadera en condiciones adversas. Con este sistema se logran altos niveles de producción animal, con la máxima utilización del forraje en su mejor estado de calidad (Pinheiro, 2004; Benítez, 1999; Senra, 2009). En este proceso resulta de gran importancia la utilización de herramientas conducentes al mejoramiento del manejo de las praderas, el Sistema de Pastoreo Racional Voisin, se fundamenta en la rotación dirigida y estratégica de los potreros buscando maximizar la producción de biomasa, el reciclaje de nutrientes, minimizar desperdicios, etc., lo que redunda en mayor producción por hectárea y mejores ingresos para el productor. Este sistema de pastoreo fue formulado en los años cincuenta por el químico y físico francés André Marcel Voisin el cual está basado en 4 leyes consideradas hoy en día pilares del manejo de pasturas.

La ley de reposo dice que para que un pasto cortado por el diente del animal pueda dar su máxima productividad entre dos cortes sucesivos, a diente, es necesario que pase el tiempo suficiente, de modo que permita al pasto almacenar en sus raíces la suficiente reserva para un comienzo de rebrote vigoroso y realizar su llamarada de crecimiento o gran producción diaria de pasto. Este período de descanso, entre dos cortes sucesivos, será variable de acuerdo a la estación del año, condiciones climáticas y demás factores ambientales. Según Voisin, (1963) El 
adecuado tiempo de reposo de la hierba es esencial para garantizar la máxima producción de biomasa con calidad suficiente para que una vez cosechada por el animal o por el órgano de corte de la segadora, sea convertida en leche, carne y otros productos con alto valor para el consumo humano, sin comprometer la persistencia del pastizal y los requerimientos nutricionales del rumiante. Otros autores reportan que un verdadero tiempo de descanso prolonga la vida de la pradera, además de permitir un aumento de la productividad de la misma, de lo contrario, ésta demora su tiempo de recuperación y envejece muy rápido (Erickson, 1976; Cuesta, 2005). Además, con el consumo de un pasto de buena calidad se logra una producción razonable de leche o incremento de peso sin deteriorar la pradera, pero esto se consigue si se respetan los tiempos de reposo, acorde con varios factores como época, especies y nivel de insumos agrícolas aplicados (Guevara y Curbelo 2006).

La ley de la ocupación indica que el tiempo global de ocupación debe ser lo suficientemente corto para que el pasto cortado a diente por el animal en el principio de ocupación, no vuelva a ser cortado por el diente del animal en el mismo período (Voisin, 1963). Aun así ha habido investigaciones que indican que altas intensidades de pastoreo o denominadas cargas instantáneas altas no provocan compactación del suelo cuando el manejo es racional; sin embargo, en situaciones de sobrepastoreo sí se produce este dañino proceso (Cuesta, 2005).

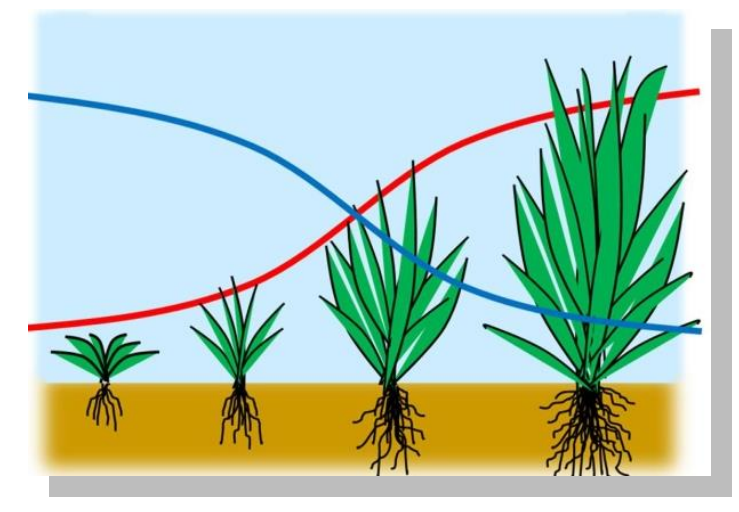

Figura 1. Relación cantidad de forraje Vs cantidad de proteína.

Fuente: Sánchez, (2009). 
El efecto posible sobre el rendimiento, calidad y persistencia del pasto que tiene el tiempo de reposo de la hierba, está muy ligado a la acumulación en las raíces y en la base de los tallos de las reservas suficientes que le permitan la formación de cierta cantidad de tejido foliar, a partir del cual comienza la fotosíntesis y se producirá el crecimiento vegetal en función del tiempo transcurrido (Brougham, 1987; Pezo e Ibrahim, 1998). Cuando el pasto es cortado, en pocos días se inicia el proceso de formación de las células verdes, comienza la fotosíntesis y la reposición de reservas en la raíz. (Pinheiro, 2004). Estas reservas son definidas como las substancias de reserva (Sorio, 2009) o sustancias orgánicas almacenadas en los órganos más permanentes de la planta para ser utilizadas como fuente de energía para el crecimiento o como material de estructura si en ese período el animal vuelve a comer la planta y si esto sucede repetidas veces motiva que se reduzca la producción de pasto (Voisin, 1963).

Es importante resaltar este tema de las reservas que existen en las raíces, porque son ellas las encargadas de alimentar la planta para que tenga un crecimiento vigoroso en ese necesario periodo de reposo, estas sustancias están divididas en dos grandes grupos: a) Carbohidratos no-fibrosos como azúcares (glucosa, fructosa, sacarosa, maltosa y otras) y polisacáridos (almidón y fructosana); b) Carbohidratos estructurales o fibrosos como la celulosa, hemicelulosa, lignina. Los carbohidratos no fibrosos son importantes fuentes de energía para las plantas, como también para los herbívoros que las ingieren. Constituyen la mayor fracción del contenido sólido del citoplasma. Los carbohidratos fibrosos constituyen la fracción más expresiva de la dieta de los rumiantes y son siempre las mayores fuentes de substrato para fermentación en el rumen. Las substancias de reserva de las plantas de las pasturas merecen atención especial. A partir de los momentos en que la planta es cortada sobra muy poco, a veces casi nada, de su parte aérea capaz de crear, por fotosíntesis, las condiciones necesarias para la formación de nuevas células y su posterior rebrote inicial. Por ese motivo resulta indispensable que la planta, al ser cortada, posea en sus raíces o en la base de 
sus tallos las substancias de reserva que le permitan rebrotar con vigor (Sorio, 2009). Al producirse un agotamiento de las reservas, como resultado de excesiva defoliación, hay una reducción en el vigor del pasto y su deterioro productivo se incrementa (Guevara y Curbelo, 2006)

La ley de rendimientos máximos o ley de las categorías o ley de la ayuda dice que se debe ayudar a los animales de exigencia alimenticia más elevada, para que puedan cosechar la mayor cantidad de pasto, y que éste sea de la mejor calidad. Cuanto menos trabajo de pastoreo a fondo se le imponga al animal, mayor es la cantidad de pasto que podrá cosechar. (Voisin, 1963) En términos prácticos, si se trabaja con categorías animales de exigencias nutricionales más elevadas (como son las hembras productoras de leche o en lactancia, los terneros en crecimiento 0 los novillos en régimen de engorde), se les debe facultar la prerrogativa de accesos diarios a pastos de calidad. (Sorio, 2009).

La ley de rendimientos regulares o ley de permanencia indica que para que un animal pueda dar rendimientos regulares, es necesario que no permanezca más de tres días en una misma parcela, y será mayor su rendimiento si no permanece más de un día. (Voisin, 1963). Se enfatiza en la necesidad de que los períodos de consumo del pasto en cada parcela sean suficientemente cortos para evitar el consumo de los rebrotes tiernos durante el mismo ciclo de pastoreo, lo que ayuda a garantizar la estabilidad del pastizal (Senra, 2009)

Es importante resaltar que el tipo de suelos de la región de los llanos orientales son ácidos y pobres en materia orgánica, lo que hace indispensable implementar sistemas de pastoreo que permitan optimizarlo, dándole un descanso a las praderas y evitando una compactación del suelo por pisoteo y sobrepastoreo. Según (Guevara y Curbelo, 2006) los suelos de relieve llano y ondulado-alomado, se caracterizan por una vegetación de sabana, laderas y bosques tropicales en menor cuantía, generalmente no poseen una gran fertilidad y además sus 
propiedades físicas e hidrológicas, su susceptibilidad a la erosión y la fertilidad natural relativamente baja, pueden influir en desbalances de nutrientes minerales.

Uno de los aspectos más importantes en el manejo intensivo es la descarga de excreciones pues propicia el incremento de la biota edáfica y de un rebrote con calidad, no obstante, su riqueza y la velocidad de descomposición es mayor cuando los árboles están formando parte del sistema. Aun el mismo creador de este sistema de pastoreo el Dr. Voisin afirmaba que, como consecuencia directa de la alta concentración de bostas y orina en el suelo, se incrementaba la biocenosis, que en este caso significa la movilización y activación biológica del suelo, con la consiguiente mejora de su fertilidad (Voisin, 1963), ahora bien, la deposición de estiércol y orina influyen en los diferentes indicadores bioquímicos del suelo. Se acepta que la bosta mejora el pH, su contenido en materia orgánica, la capacidad de intercambio catiónico y los tenores de algunos microelementos minerales (Primavessi, 1990).

También es importante el factor suelo en donde van a quedar todas estas deyecciones como la bosta y la orina, la cual será procesada por un sin número de organismos encargados de reincorporar los nutrientes al suelo (Estrada, 2001). Así como lo resaltan Kolmans y Vázquez (1996), citados por Sánchez et al. (2009), tomando al suelo como base de cualquier sistema de explotación destacando que un metro cuadrado de suelo vivo contiene aproximadamente 10 millones de nematodos, 100 mil colémbolos, 45 mil anélidos y unos 40.000 insectos y ácaros, así mismo un gramo de suelo contiene 500 mil bacterias, 400 mil hongos, 50 mil algas y unos 30 mil protozoarios aproximadamente. Todos estos microorganismos son mejoradores del suelo (Arias, 2007; Lascano y Spain 1991)

Es importante resaltar la importancia que tienen los arboles en los sistemas de pastoreo ya que cumplen funciones como incorporadores de nutrientes al suelo, fijadores de nitrógeno atmosférico, brindan bienestar animal por el aporte de la 
sombra y como alimento en el caso de ramoneo cuando la disposición de los arboles es para tal fin. Esto coloca al árbol como un factor estratégico de manejo, determinante en la sostenibilidad y eficiencia de los pastizales en el trópico estacional, ya que mejora la cobertura vegetal contra la erosión pluvial, aporte de materia orgánica al suelo, como de alimento animal, y en disminuir la evaporación y el escurrimiento, que es superior en los pastizales desprovistos de arboles. (Pezo e lbrahim, 1998).

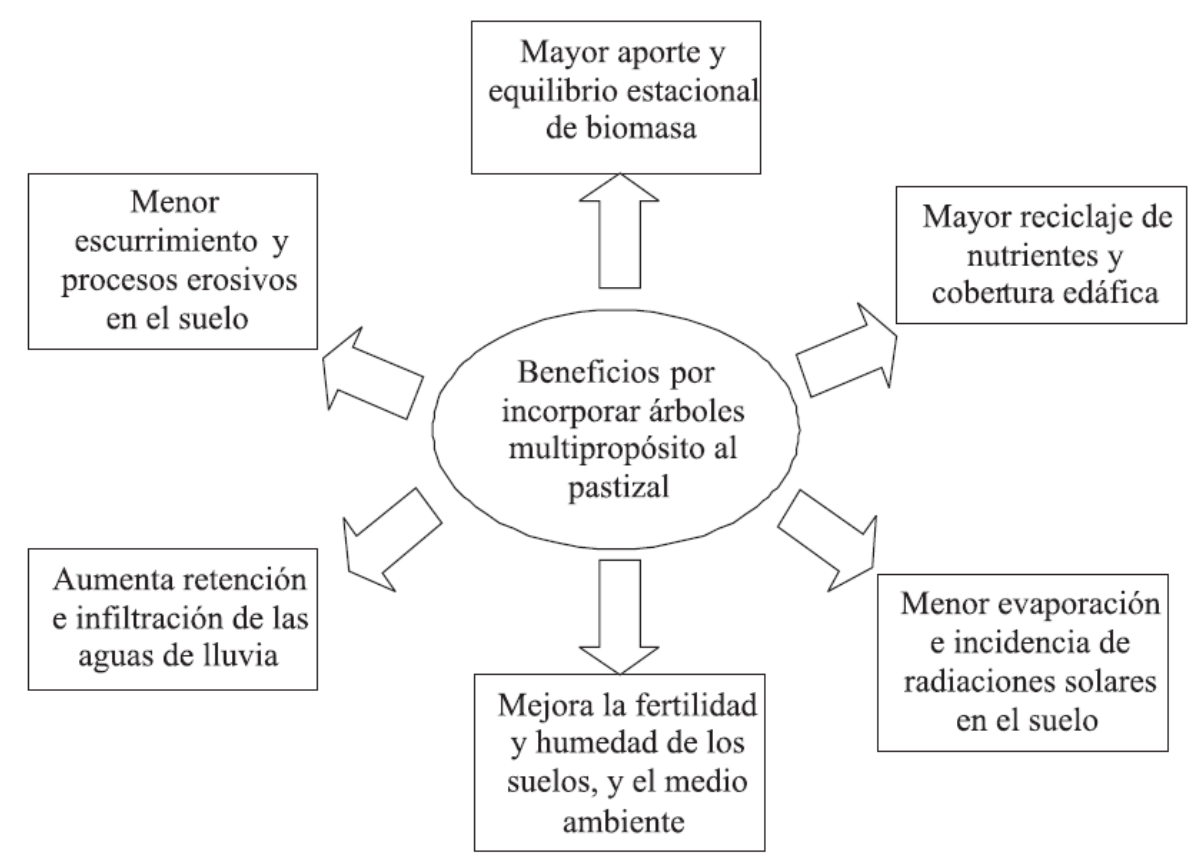

Figura 2. Beneficios adicionales de la estrategia de manejo de incorporar los árboles multipropósito al pastizal (silvopastoreo). Fuente: Senra, (2009)

Se acepta que el mal manejo del ecosistema del pastizal es una de las causas fundamentales de la degradación de los pastos y el suelo, lo que conduce a una baja eficiencia en los sistemas de explotación de bovinos basados en pastoreo, que incluye la insostenibilidad del rebaño (Senra, 2009). Según Cuesta (2005) se considera que un pasto está degradado cuando la especie deseable pierde su vigor y capacidad productiva por unidad de área y por animal, la cual se reemplaza por especies de escaso rendimiento y valor nutritivo, así como áreas despobladas. 
Además hay cambios en el microclima del suelo, que se manifiesta por un aumento de la temperatura y una disminución de la humedad. De acuerdo con Renda (2006), en la ganadería, cuando se degradan los suelos por compactación de la capa superior, el nivel de materia orgánica se reduce y se destruye la estructura y su estabilidad, ya que favorece el escurrimiento superficial y, consecuentemente, los procesos erosivos, lo que hace insostenible la producción ganadera. Por esta razón los sistemas silvopastoriles son una alternativa para mejorar suelos y ayudar a la reconversión ganadera (Pezo e Ibrahim, 1998).

La capacidad de carga define la cantidad y tipo de animales que pueden permanecer en una pradera sin alterar su sostenibilidad. Suele darse en unidades grandes de ganado (U.G.G.) o en kilogramos totales por unidad de área. La Unidad Gran Ganado se entiende como el peso de un animal adulto. En el caso de ganado de leche especializado equivale a $650 \mathrm{~kg}$; en ganado de doble propósito, una vaca con su cría, lo cual se estima en $450 \mathrm{~kg}$. Para realizar los cálculos, se da al toro el valor de $1.6 \mathrm{UGG}, 1.0$ a la vaca horra, 0.8 a la novilla de vientre, 0.6 la de levante y 0.2 para las crías.

\section{OBJETIVOS}

- Implementar el sistema de pastoreo racional (sistema Voisin) en la finca la Gloria en el municipio de puerto López (Meta).

- Hacer un diagnóstico inicial para conocer el estado de la finca y como se encuentra en términos de suelo, forraje que se ofrece al ganado, fuentes de agua y demás recursos que permitan determinar en qué condiciones se ha de ejecutar el proyecto.

-Planificar con base en el diagnóstico inicial cuales son las mejores condiciones para la realización del proyecto, al igual, que un presupuesto de acuerdo a lo aprobado entre las partes interesadas en la ejecución. 
-Ejecutar el proyecto de implementación del sistema de pastoreo racional Voisin de acuerdo a lo establecido y aprobado por parte del productor

-Organizar el sistema de pastoreo actual para ayudar en mejoramiento de los índices de producción (ganancia de peso) en el ganado bovino de la finca la Gloria en la vereda Melúa municipio de Puerto López en el departamento del Meta.

-Concientizar a los empleados de la finca la Gloria en buenas prácticas ganaderas, biosanitaria y lo relacionado con el correcto manejo de las praderas.

-Acompañar otras actividades medico-veterinarias y labores propias de la finca que se dieron durante la estadía y la ejecución del proyecto

\section{ACTIVIDADES REALIZADAS}

Las actividades realizadas se dividieron en cuatro partes: Diagnóstico, planificación, presupuesto y ejecución del proyecto. Inicialmente se hizo una visita de caracterización en la cual se consignaron datos como medidas de la finca, población total de animales, tipo de forrajes que se emplean, estado sanitario, fuentes de agua, etc. Luego de esta visita de caracterización se inició un plan de mejoramiento, esto era parte de un trabajo que se hacía en conjunto con el Comité de Ganaderos del Meta, sin embargo, el interés principal fue el montaje del Sistema de Pastoreo Racional Voisin, aun así, todos estos datos sirvieron de base para planear, presupuestar y ejecutar el proyecto.

En las visitas de caracterización realizadas a la finca se recorrió todo el terreno con el objeto de conocerlo y observar las áreas más idóneas para la implementación del sistema de pastoreo, fue así como se visitaron todos los potreros, fuentes de agua, corrales, entre otros. Durante el recorrido se formularon preguntas relacionadas con el manejo de animales, praderas, corrales, estado sanitario y de bioseguridad, de tal manera, que con la información suministrada se pudo formular un plan de mejoramiento, para ajustar e implementar el proyecto en la finca La Gloria. Este diagnóstico se inició con el recorrido de los potreros donde 
se encontraron áreas muy degradadas por el sobrepastoreo y pisoteo de los animales, terrenos con drenaje regular, áreas de pastoreo muy extensas lo que permitía que los animales pudieran recorrerlo todo sin ningún tipo de restricción, práctica que es discutida por Castro, (1999) destacando que es una de las causales de degradación de las praderas, por lo tanto, representaba una pérdida de forraje, que no sería consumido, provocando una acumulación de biomasa, y pisoteo excesivo de la pradera por parte de los animales. Según información previa de la finca, el tiempo de ocupación de los potreros estaba en el orden de los 25 a 30 días, mientras que el periodo de descanso era de 2.5 a 4 meses, es así, como se puede deducir que por un periodo de ocupación tan extenso, estuviera provocando un deterioro de la pradera, lo que a su vez, hacía que el periodo de recuperación del pasto fuera prolongado. Todo esto facilitaba que el ganado consumiera los rebrotes en varias ocasiones antes de ser cambiados de potrero o antes de cumplir su periodo de ocupación lo que es perjudicial puesto que mientras más joven es consumido el pasto, menos productiva se hace la pastura y menor capacidad de carga puede soportar, y tan sólo el hecho de permitir que los animales permanecieran por un largo tiempo en el mismo potrero y consumiera los rebrotes jóvenes, le causaba al pasto un efecto de enanismo (acortamiento entre los nudos de la planta) al no permitirle desarrollarse totalmente hasta su punto de cosecha (Rua, 2009).

La mayoría de los potreros eran extensos variando desde las 30 ha, hasta 70 ha, lo cual generaba pérdidas considerables de forraje, bajo reciclaje de nutrientes, degradación del suelo por pisoteo, recuperación tardía de la pradera y por consiguiente, bajos índices de producción y pérdidas económicas para la explotación. Es conocido que cuando hay áreas grandes de pastoreo el ganado tiene facilidad para desplazarse y crear según Pereyra y Leiras, (1991) citado por Díaz, (2007) "manchones" o áreas de degradación parcial, pues el recorrido es mayor cuanto mayor sea el área de pastoreo, en muchas ocasiones la mala calidad del forraje hace que estos recorridos se acrecienten en busca de mejores 
pasturas, y todo esto sumado ocasiona un mayor índice de degradación de la pradera (Bignoli, 1971, citado por Díaz, 2007).

Las especies forrajeras empleadas en la finca son Brachiaria humidicola (Pasto dulce), Brachiaria decumbens (Pasto amargo), Brachiaria humidicola cv dyctioneura (Pasto llanero) y Panicum maximum (Pasto Mombaza), acompañado de pasto nativo (Eleusine indica). Las fuentes de agua para los animales en los potreros eran variables, por ejemplo, en algunos, cruzaba un caño cercano (Caño Yucao) o había un nacedero dentro de la misma área de pastoreo, no obstante, habían potreros en los que no contaban con una fuente de agua lo que obligaba a los animales a recorrer largas distancias para obtener el liquido lo cual genera un stress calórico, que a su vez ocasiona un gasto energético por dos razones una en el esfuerzo físico y el segundo por termorregulación. Por otra parte, en época de verano, se tenían que abrir varios broches convirtiéndose en un pastoreo continuo de modo que el ganado pasara de potrero en potrero hasta buscar una fuente de agua, lo que hacía que el pisoteo fuera constante en determinadas áreas creando callejones por donde siempre era pisoteado, estas características muestran lo precario que era el suministro del agua para estos animales en época de sequía.

El manejo de los animales en los corrales se dificultaba debido a las áreas tan grandes para guiar a los vacunos. Además el suelo es rocoso (Figura 3), lo que provocaba un alto riesgo de accidente para los animales, como fractura de huesos o contusiones. El trato por parte de los trabajadores no era el indicado, se usaban gritos, leños y tábanos, además había presencia de animales extraños para los vacunos (perros). Todos estos factores van en contra de las buenas prácticas ganaderas lo que genera estrés en los animales y la producción se ve afectada.

Posterior al diagnóstico realizado en los potreros, fuentes de agua y manejo de los animales, factores de gran importancia para planear y ejecutar el proyecto, se inició un recorrido para encontrar el terreno adecuado y así poder implementar el 
sistema de pastoreo. Dentro de las labores realizadas están la selección, medición, diseño de mapas y modificación de las áreas de pastoreo entre otras.

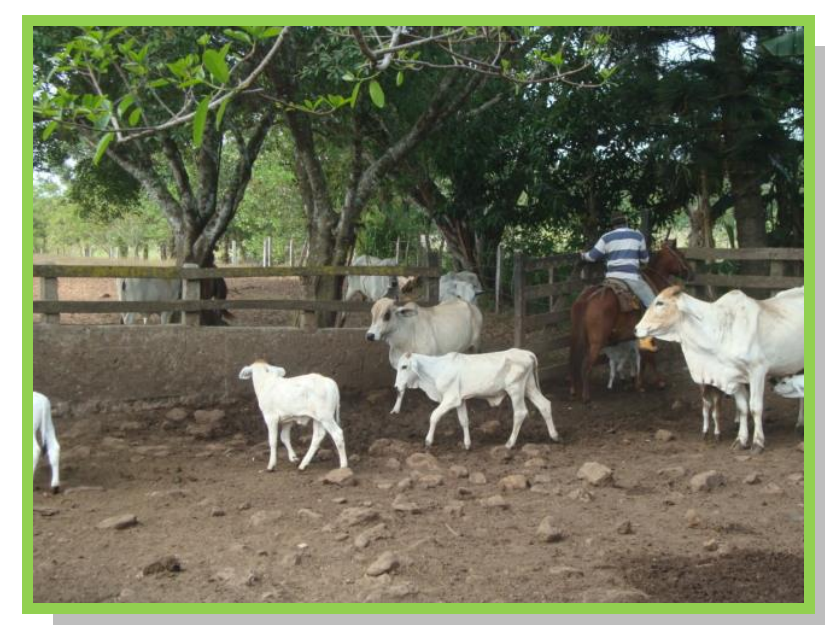

Figura 3. Condiciones del corral

En el proceso de selección del área para la realización del sistema de pastoreo, se recorrieron los terrenos de la finca para establecer el lugar que sería indicado para la ejecución del proyecto. Teniendo en cuenta factores como fuentes de agua, áreas de sombrío, drenaje del terreno, tipo de suelo, topografía, entre otros. La medida del área de trabajo se realizó por medio de un dispositivo de medición satelital (GPS), que sirvió de soporte para diseñar los potreros. El resultado del registro de medición del GPS fue de 64 hectáreas, con base en estas medidas se obtuvo otras que indicaban las áreas no productivas, es decir, que no contaba con pasto ya que son rocosas. Posteriormente, se diseñaron las áreas de pastoreo, teniendo en cuenta los callejones centrales y auxiliares para el traslado de los animales sin tener que ocupar potreros en dicho recorrido.

Con la medida total del área de trabajo se inició el diseño de los potreros y de sus callejones. En un principio, los planos recomendados fueron de 2 hectáreas por cada potrero, para lo cual, se presupuestó con dichas medidas, aun así, la administración de la finca solicitó que se plantearan potreros de mayor tamaño, 
por lo tanto, se rediseñaron potreros de 4 hectáreas para un total de 14 potreros, que fueron de gran aceptación y fueron aprobados.

Durante el recorrido para tomar las medidas con el dispositivo de medición satelital GPS de toda el área que posteriormente se subdividiría para adecuar las áreas de pastoreo, se encontró que el terreno no era totalmente cuadrado o rectangular (Figura 4) así como se desearía que fueran, para facilitar el diseño de los potreros y de los callejones. Esto hizo interesante el diseño de los potreros puesto que fueron necesarias varias propuestas para decidir finalmente cual sería la disposición de cada área de pastoreo, se buscó que fueran lo más uniforme posible. En cada diseño se modificaron ubicación de bebederos, sistema hidráulico, callejones, entre otros. (Figuras 5).

Figura 4. Plano del área de trabajo. Las áreas de color café indican la parte rocosa o no productiva que había en el terreno; el verde más oscuro es el área boscosa o mata de monte; la franja verde con la línea curva de azul indica un caño veranero que pasaba cercano (Caño Yucaito), el color verde claro es toda el área productiva sembrada en pasto Brachiaria humidicola.

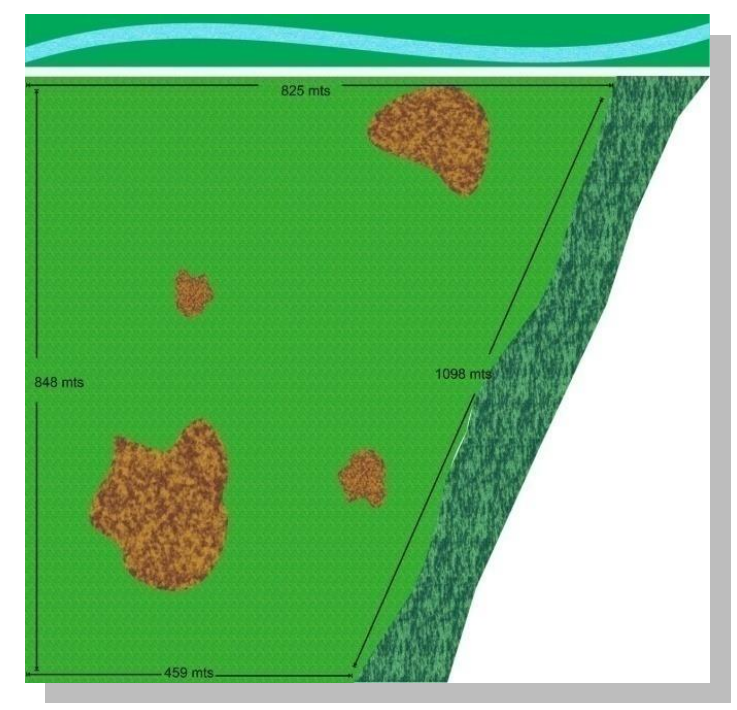

Figura 4. Plano del área de trabajo.

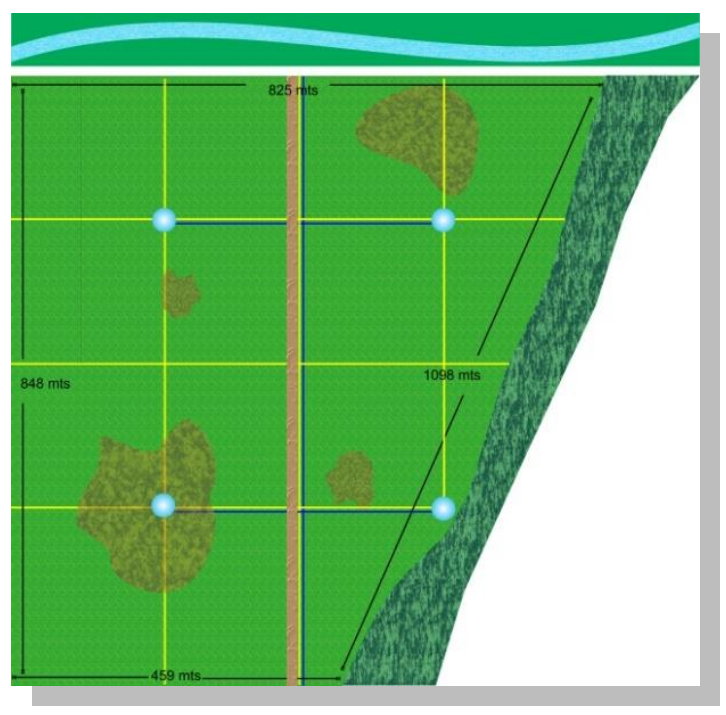

Figura 5. Plano definitivo del proyecto 
La planificación y el presupuesto que fueron aprobados para la implementación del sistema de pastoreo racional Voisin permitieron la adecuación de los potreros, con lo cual se promedio un gasto de $\$ 600.000$ por hectárea en sistema hidráulico y un promedio de $\$ 300.000$ por kilometro de cerca eléctrica, posteriormente se ingresaron los animales y se inició la evaluación de las ganancias de peso y el crecimiento del pasto.

Con los planos de los diseños de cada uno de los potreros y con el conocimiento pleno de los puntos exactos de donde iría cada elemento como postes, cuerda de alambre, sistema hidráulico, y demás elementos, se procedió a la ejecución del proyecto, para lo cual, se tuvo en cuenta los lugares donde quedarían ubicados los callejones mencionados anteriormente, los bebederos, áreas sociales, saladeros, entre otros.

El recurso hídrico es quizás considerado el factor más importante en el montaje de este tipo de sistema (Castro, 1999; Sorio, 2009). Por lo tanto, para el abastecimiento del agua fue necesario hacer un pozo de 35 metros de profundidad, del cual se bombeaba el agua hacia un tanque que se encontraba en la parte más alta del terreno de trabajo hecho en concreto y sobre tierra con una capacidad de 14.000 litros ubicado en uno de los lugares más altos de la finca. Por una de las salidas del tanque se extendió un tubo madre que llegaba hasta el potrero, posteriormente, se ramificaba hasta llegar a los bebederos que abastecía por gravedad a cada uno de los potreros, los cuales eran tanques de capacidad de 1.000 litros.

Las líneas eléctricas más cercanas pasan a 4 kilómetros de allí, por lo que se hacía muy costoso traer por postes elevados a través de esa distancia hasta la finca y así poder tener fluido eléctrico, de tal manera, que para abastecerse de energía eléctrica se recurrió a un sistema de energía solar, mediante paneles que toman la energía solar para transformarla en eléctrica, para lo cual, se utilizaron 2 paneles que fueron instalados estratégicamente en un sitio húmedo del área de 
trabajo. De esta manera, se pudo abastecer el flujo eléctrico que requerían las cercas.

Se realizaron aforos en cada uno de los potreros establecidos para hacer los cálculos que permitiera conocer la cantidad aproximada de forraje y asimismo, establecer la carga animal. Se inició el pastoreo con el ingreso de 60 bovinos machos de $350 \mathrm{~kg}$ de peso aproximado (1.4 UGG/ha), los cuales fueron rotados por todo el sistema con un periodo de ocupación en cada potrero de 4 días; esta cantidad de animales se escogió gracias al cálculo obtenido de los aforos realizados en cada potrero y se estimó una capacidad de carga de 1 a 1.5 UGG/ha. Cabe aclarar que como cada potrero tenía aproximadamente una medida de 4 ha, se dejaban allí en pastoreo por un lapso de 4 días, es decir, 1 día de ocupación por hectárea, dentro de cada potrero se franjeaba para garantizar que se consumiera el forraje de todo el potrero y que no fuera pisado el área pastoreada del día anterior. De la misma manera, se rotaban al siguiente potrero con un tiempo de ocupación similar al anterior, hasta completar el recorrido por todo el sistema. Para el segundo mes se duplico la carga animal por lo cual se llegó a 2.8 UGG/ha y para el tercer mes se logró tener $4.1 \mathrm{UGG} /$ ha puesto que ya había 150 animales de $400 \mathrm{~kg}$ aproximadamente.

El área social conocida como el área donde los animales están en las horas pico del día en donde se presenta más radiación solar (10:00 AM - 4.00 PM), allí se dedican a hacer la rumia y tienen a voluntad agua y sal, además, están bajo sombra pues es uno de los requisitos para una verdadera área social pues se está buscando minimizar estrés en lo animales por calor y por esfuerzo físico en búsqueda de agua. Aun así, hasta el momento de la finalización del proyecto aun no se había podido adecuar un área social definida ya que se presentó dificultades administrativas que no dieron vía libre para la ejecución de dicha área, aun así, en cada potrero se contaba con árboles que mitigaban el efecto del calor en las horas pico de mayor radiación solar (10:00 am -4:00 pm). Además, se realizaron 
jornadas de corte de pasto Kingrass para hacer ensilaje junto con botón de oro en un porcentaje de $75 \%$ de gramíneas y un $25 \%$ de botón de oro (Figura 7 .)
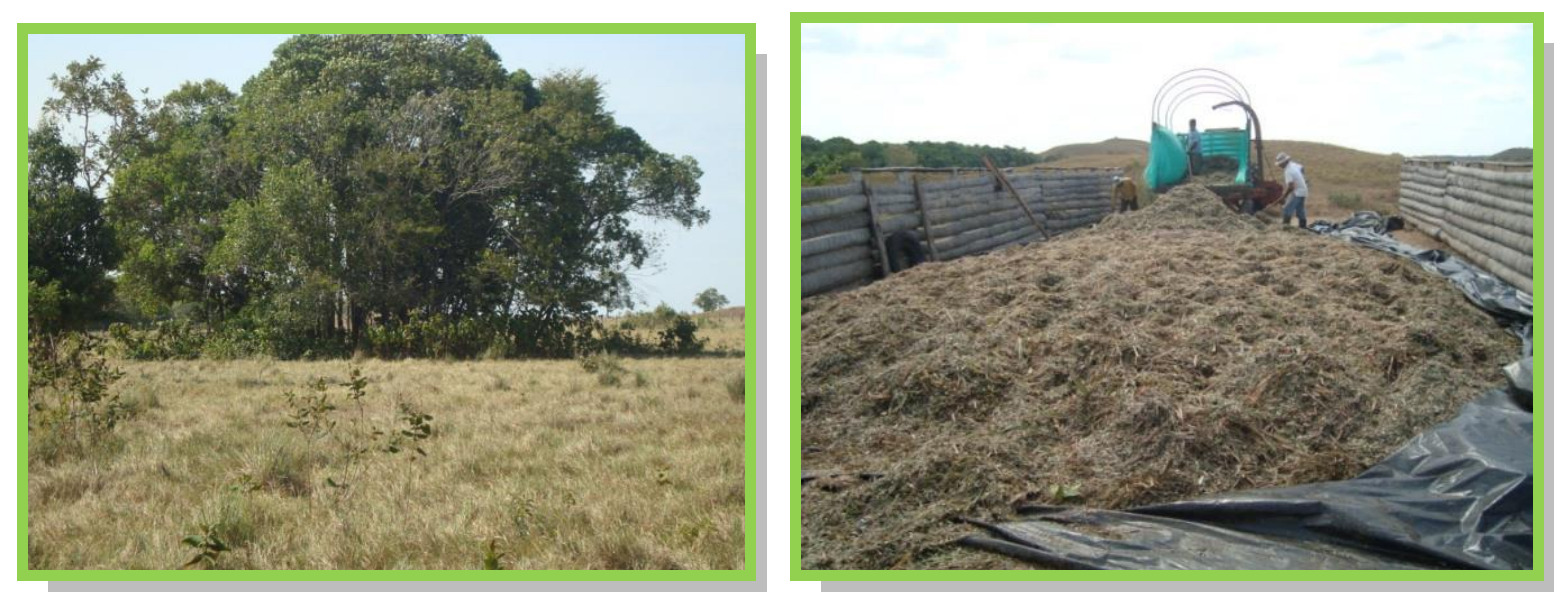

Figura 6. Área social y arborización en potreros Figura 7. Preparación de ensilaje

Constantemente se estaba interactuando con los trabajadores, resaltando la importancia de la ejecución y el mantenimiento del sistema de pastoreo racional en la finca, el manejo de los animales teniendo en cuenta su bienestar, el uso adecuado de los medicamentos, y todo lo relacionado con las buenas prácticas ganaderas y el manejo sanitario. Esto se hizo con el ánimo de mantener el sistema de pastoreo en la finca y que se retroalimentaran las experiencias encontradas en el manejo de dicho sistema y asimismo, hacer los correctivos necesarios, buscando que este tipo de sistema se fuera implementando en toda la finca, y de alguna manera, fortalecer la mano de obra para que esta fuera calificada por el conocimiento de este tipo de pastoreo racional como alternativa para incrementar la productividad de la finca (Rua, 2009).

\section{RESULTADOS Y DISCUSIÓN}

La rotación fue uno de los factores más cambiantes en todo el proceso del trabajo realizado en la finca, puesto que la recuperación del forraje en cada potrero fue independiente y mostró mejores resultados comparativos entre un área de otra, (Figura 7) lo que a su vez, permitió el traslado de los animales de una manera 
aleatoria y no consecutiva. Es importante resaltar que este tipo de sistema de pastoreo en función del traslado estratégico de los animales permite darle un mejoramiento al suelo por las deyecciones de bosta y orina que se depositan, propiciando el incremento de la biota edáfica y de un rebrote con calidad, entre otros beneficios (Primavessi, 1990).

Una consecuencia directa de esta concentración de bosta y orina en el suelo, es el incremento de la biocenosis, que en este caso significa la movilización y activación biológica del suelo, con la consiguiente mejora de su fertilidad (Voisin, 1963). Además de mejorar el $\mathrm{pH}$, su contenido en materia orgánica, la capacidad de intercambio catiónico y los tenores de algunos microelementos minerales se incrementa.

A partir del primer mes cuando se inició la rotación de los animales por cada uno de los potreros, se empezó a observar áreas que se recuperaban más rápido que otras y zonas más aprovechadas por el ganado, de tal manera, que antes de iniciar el segundo ciclo de rotación las cargas animales se modificaron pasando de 1.4 a 2.8 U.G.G./Ha, es decir, de 60 a 120 animales respectivamente, puesto que este sistema consiste en no darles una rotación secuencial sino racional (Sorio, 2009), es decir, dependiendo de las condiciones del forraje y de su punto óptimo de reposo (POR).

Lo expuesto anteriormente se dio principalmente por las condiciones del terreno, pues había una parte alta y una baja. Según información previa, la zona baja no era tan pastoreada por el ganado cuando se le dejaba allí, lo que favoreció la recuperación de esta parte en comparación con las zonas altas, en donde si era muy pastoreada por los animales (Figura 8) este proceso fue mencionado por Pereyra y Leiras, (1991) citado por Díaz, (2007). 

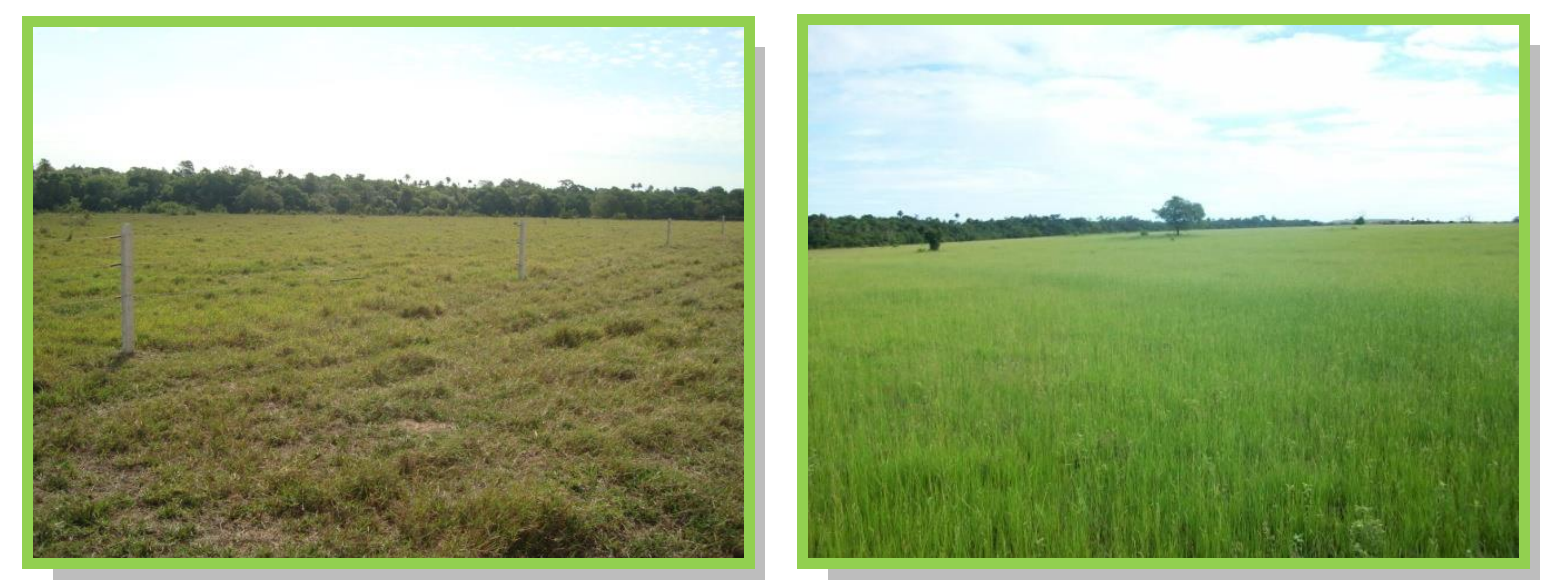

Figura 7. Vista de potreros en rotación. Figura 8. Recuperación regular de potreros

Durante la rotación por cada uno de los potreros se hicieron mediciones del forraje para conocer su recuperación en términos de tiempo (Tabla 1).

Tabla 1. Crecimiento del forraje en ciclos de rotación

\begin{tabular}{ccc}
\hline \multicolumn{3}{c}{ Crecimiento del forraje (cm) } \\
\hline \# Rotación & $\mathbf{1}$ & $\mathbf{2}$ \\
\hline 1 & 1 & 1 \\
2 & 1.5 & 1.6 \\
3 & 3 & 2.8 \\
4 & 4 & 4.1 \\
5 & 5 & 5.3 \\
6 & 4 & 4.2 \\
7 & 2 & 2.5 \\
\hline
\end{tabular}

La oferta forrajera con la que se contó al inicio de la implementación del sistema de pastoreo racional Voisin fue de 3.4 ton/ha aproximadamente, resultado que se da luego de restarle el $30 \%$ que se pierde por pisoteo. Por lo que se inició con una carga animal de $1.4 \mathrm{UGG} / \mathrm{ha}$, ahora bien, esta misma oferta para el segundo ciclo de rotación fue de 4.2 Ton/ha lo que permitió aumentar la carga animal de 1.4 a 2.8 UGG/ha con un periodo promedio de descanso de 45 días, gracias a que la recuperación del forraje fue mejorando significativamente durante el primer ciclo de rotación. En un periodo anterior y bajo el sistema tradicional esta cantidad de animales hubiera sido insostenible (Estrada, 2001; Castro, 1999). 
Aunque se reporta carga animal de hasta 6 animales/ha con éste sistema de manejo (Pinheiro, 2004), debemos considerar que en zonas como la altillanura, con suelos ácidos y con baja calidad de pasturas no es fácil pretender llegar a esta carga animal, además, es prudente iniciar con una carga animal baja y con la ayuda de un buen manejo determinar si es posible ir aumentando progresivamente, lo que se dará con el mejoramiento de la pradera y la respuesta del forraje ante el manejo que se establezca. No obstante, para nuestras condiciones edafoclimaticas los resultados que se obtuvieron son importantes, y se podrían mejorar. Lo importante es encontrar las estrategias para conseguirlo y se tiene la certeza que este sistema de pastoreo racional es uno de los que mejor ofrece esta posibilidad y no queda duda que se puede aplicar en la altillanura con buenos resultados.

En el pesaje de los 12 animales (20\% de la población empleada en el pastoreo) los cuales fueron evaluados durante los dos ciclos de rotación que se lograron durante el trabajo realizado en la finca, se compararon con animales de la misma edad en diferentes condiciones de pastoreo, encontrándose resultados de ganancias de peso entre 120-190 g/día más que los animales que estaban bajo el sistema tradicional teniendo en cuenta que el promedio de ganancia de peso de la finca es de $510 \mathrm{~g} / \mathrm{día}$.

Según registros de la finca en donde se indicaba el promedio de ganancia diaria de peso éste reportó pesos entre 480 y $515 \mathrm{~g} /$ día en pastoreo tradicional, por lo tanto, bajo el sistema de pastoreo racional Voisin se tomaron 10 animales y se pesaron inicialmente y luego cada mes para evaluar ganancias bajo dicho sistema y comparar finalmente con el tradicional. Los animales bajo el sistema de pastoreo Voisin tuvieron ganancias de peso entre 533 y 700 g/día, con un promedio de 630 gr/día, lo que permite afirmar que superaron el promedio de ganancia diaria de peso en la finca, mejorando así uno de los índices productivos, que en ultimas, se ve reflejado en mas carne por unidad de área. 
Tabla 2. Pesos de animales en prueba de pastoreo

\begin{tabular}{ccccc}
\hline \multirow{2}{*}{ Numero } & \multicolumn{4}{c}{ Pesos de los animales bajo sistema de pastoreo Voisin (kg) } \\
\cline { 2 - 5 } & Antes & 1er mes & 2do mes & 3er mes \\
\hline 1 & 348 & 368 & 390 & 411 \\
2 & 355 & 372 & 391 & 409 \\
3 & 352 & 370 & 390 & 405 \\
4 & 355 & 368 & 388 & 407 \\
5 & 360 & 375 & 396 & 408 \\
6 & 349 & 367 & 389 & 410 \\
7 & 352 & 373 & 394 & 413 \\
8 & 350 & 365 & 388 & 410 \\
9 & 352 & 371 & 392 & 415 \\
10 & 352 & 369 & 389 & 408 \\
11 & 350 & 372 & 390 & 409 \\
12 & 355 & 370 & 390 & 406 \\
\hline
\end{tabular}

Los índices productivos de la finca fueron mejorados con estas ganancias de peso, además, como se dio un uso correcto a las áreas de pastoreo, esto permitió un verdadero descanso a las praderas por lo que su recuperación fue satisfactoria y a su vez, se pudo aumentar la carga animal por hectárea, lo cual infirió en la productividad de la finca. Entre otras cosas se pasó de producir en 64 hectáreas 21 toneladas de carne a 52 toneladas en una misma área, es decir, 0.3 toneladas de carne por hectárea a 0.8 toneladas de carne por hectárea.

La enseñanza del manejo sanitario y de las buenas prácticas ganaderas fue y es un tema de difícil acogida en las ganaderías tradicionales las acciones a veces se encuentran amparadas bajo las frases como "Aquí siempre se ha hecho así" y "como lo hacemos nunca ha pasado nada", aun así, bajo un trabajo arduo y con demostraciones se logró cambiar en gran parte la cultura en la parte del manejo sanitario. Dentro de estas acciones estuvo el de lavado periódico de los corrales, uso de una aguja por animal, lavado de bretes y de bascula, almacenamiento correcto de los medicamentos, revisando vencimientos, dosis recomendadas entre 
otros, logrando una buena aceptación por parte de los trabajadores en la mayoría de los casos, todo esto visto en conjunto con las buenas prácticas ganaderas que también contempla y da mucha importancia al bienestar animal.

\section{CONCLUSIONES}

La implementación del sistema de pastoreo racional Voisin es indicado para contribuir al mejoramiento de los índices de producción, ya que permitió pasar de producir en 64 hectáreas 21 toneladas de carne a 52 toneladas en una misma área, es decir, 0.3 toneladas de carne por hectárea a 0.8 toneladas de carne por hectárea, teniendo en cuenta que el uso correcto de praderas es determinante para que se dé un punto óptimo de reposo, de tal manera, que podemos producir mayor cantidad de carne por unidad de área.

Con el pastoreo racional se logra definitivamente un mejoramiento de la calidad del forraje y se acortan los tiempos, logrando así ganancias de hasta $0.5 \mathrm{~cm}$ mas de tamaño foliar en el mismo tiempo de reposo empleado anteriormente, lo que permite dar un ciclo más corto sin dejar de ser productivo, puesto que lo determina también la cantidad de forraje producido y asimismo, el reajuste de la carga animal.

Es importante la realización de los aforos mínimo 2 veces por semana, y de esta manera conocer cantidad de forraje que se tiene para ofertar, al igual que el ajuste de la carga animal para cada potrero.

\section{RECOMENDACIONES}

Realizar un programa de arborización para crear otras áreas sociales o en su defecto arborizar cada uno de los potreros para garantizar sombra a los animales durante las horas de mayor radiación solar, ya que es indispensable para crear confort en los animales que hacen uso del sistema de pastoreo racional Voisin lo cual permitiría a su vez un mayor consumo de forraje, mejor conversión alimenticia y un aumento en la ganancia de peso. 
Implementar una subdivisión estratégica de los potreros, de tal manera, que queden más reducidos, es decir, a una hectárea preferiblemente, con lo cual, se pueda manejar mejor los animales, además la rotación se hará de manera más sencilla sin tener que franjear cada potrero como se ha hecho hasta el momento.

Tener un adecuado manejo en la rotación de los potreros para evitar pérdidas de forraje o acumulación de biomasa lo cual generará pérdidas económicas traducidas en bajo consumo de forraje, menor ganancia de peso y si el caso es contario y se hace mayor presión de pastoreo aumentaría el pisoteo y conllevaría a una degradación del suelo que sería el peor de los casos.

Realizar los aforos permanentemente, para así poder hacer los ajustes necesarios y observar en cuanto se puede o no aumentar la carga animal en cada potrero que se vaya a utilizar, respetando las leyes del animal y de los pastos como lo contempla y recomienda el sistema de pastoreo rotacional.

Examinar cuidadosamente la parte biosanitaria y que se cumpla a cabalidad para que no se presenten problemas de salud en animales que estando sanos se puedan llegar a contagiar de los enfermos por un factor iatrogénico, en especial con el uso de las jeringas. De igual manera hacer planes de medicina preventiva.

Seguir generando en los trabajadores el sentido de pertenencia por el uso de este sistema de pastoreo y hacer un proceso de retroalimentación para así conocer las fortalezas y debilidades del sistema, de tal manera, que cada día se pueda mejorar los índices de producción ganadera de la finca, que es el propósito final de toda explotación pecuaria y en especial la ganadera.

\section{BIBLIOGRAFÍA}

1. Arias, A. Suelos Tropicales. San Jose: Universidad Estatal a Distancia, 2007.

2. Arteaga, O.; Frometa F. C. Los pastos como base alimentaria del ganado bovino en las condiciones actuales del Escambray. Resumen Seminario Cientifico Técnico Estación Experimental de Suelos y Fertilizantes Escambray, Las Villas, 1997. 
3. Benítez, D. Adecuación de la tecnología cubana de producción ganadera bajo el pastoreo racional a las características del Valle del Cauto. Informe de Trabajo PNTC, La Habana, 1999.

4. Brougham, R. W. Sistemas Prácticos Ganado - Forrajes. New Zeland Agricultural Science (services LTD) 21-26 1987.

5. Castro, A. Producción Bovina. Tercera reimpresión. San José: Universidad Estatal a Distancia, 1999.

6. Cuesta, P. Producción y utilización de recursos forrajeros en sistemas de producción bovina de las regiones y valles interandinas. Bogotá: Corpoica, 2005.

7. Díaz, R. O. Utilización de pastizales naturales. Editorial Brujas. Córdoba, Argentina. 2007.

8. Erickson, A. Editado por A Erickson. Boletín de Centro Agronómico Tropical de Investigación y Enseñanza (CATIE) IV, № 1: 2-5. 1976

9. Estrada, J. Pastos y forrajes para el trópico colombiano. Segunda edición. Manizales: Universidad de Caldas, 2001.

10. Guevara, G. R.; Curbelo, L. Pastoreo Racional Voisin para la producción bovina sostenible. 2006. Disponible en: http://www.reduc.edu.cu/147/04/1/14704101.pdf (último acceso: 20 de Febrero de 2011).

11. Lascano, C.; Spain, J. Establecimiento y Renovación de Pasturas. Conceptos, Experiencias y enfoque de la Investigación. Editado por México Memorias Red Internacional de Evaluación de Pastos Tropicales Veracruz. Cali: CIAT, 1991.

12. Milera, M. Fundamentos de manejo y utilización de pastizales. Disponible en: http://mvz.unipaz.edu.co/textos/lecturas/pastos-y-forrajes/fundamentos-de-pastos-yforrajes/lecturas/pastos/fundamentos-de-manejo-y-utilizacion-de-pastizales.pdf (último acceso: 25 de Febrero de 2011).

13. Pezo, D.; Ibrahim M. Sistemas Silvopastoriles. Turrialba: CATIE Proyecto agroforestal. 1998.

14. Pinheiro, L. C. Pastoreo Racional Voisin: Tecnología agroecológica para el Tercer Milenio. Buenos Aires: Hemisferio Sur. 2004.

15. Primavessi, A. Manejo Ecológico de Suelo. Agricultura en Regiones Tropicales. Sao Paulo: Libreria Nobel. 1990.

16. Renda, A. Papel de los Sistemas agroforestales en el escenario agrario de las cuencas hidrográficas de Cuba. Pastos y Forrajes, p 29-35. 2006.

17. Rua, M. Manual Ilustrado de Confinamiento Bovino. Cultura Empresarial Ganadera, 2009.

18. Sánchez, S., Crespo, G.; Hernández M. Descomposición de la hojarasca en un sistema silvopastoril de Panicum maximum y Leucaena leucocephala (Lam) de Wit cv. Cunningham. II Influencia de los factores climáticos. Pastos y Forrajes, 32 (4). 2009.

19. Senra, A. Impacto del manejo del ecosistema del pastizal en la fertilidad natural y sostenibilidad del suelo. Avances en Investigación Agropecuaria, 13 (2): 3-15. 2009. Disponible en: http://www.ucol.mx/revaia/anteriores/PDF\%20DE\%20REVISTA/2009/mayo/1\%20Imp acto\%20del\%20manejo\%20(Dr.\%20Senra).pdf (último acceso: 22 de Febrero de 2011).

20. Sorio, H. Pastoreo Voisin: Teorías- Practicas- vivencias. 2. Passo Fundo: Méritos, 2009.

21. Voisin, A. Productividad de la hierba. España: Tecnos S.A, 1963. 